\title{
The star cluster population of NGC $5253^{\star}$
}

\author{
G. Cresci $^{1}$, L. Vanzi ${ }^{2}$, and M. Sauvage ${ }^{3}$ \\ 1 Dipartimento di Astronomia, Universitá di Firenze, Largo E. Fermi 5, 50125 Firenze, Italy \\ e-mail: gcresci@arcetri.astro.it \\ 2 ESO, Alonso de Cordova 3107, Casilla 19, Santiago 19001, Chile \\ 3 Service d'Astrophysique, CEA/DSM/DAPNIA, Centre d'Études de Saclay, 91191 Gif-sur-Yvette Cedex, France
}

Received 5 April 2004 / Accepted 9 November 2004

\begin{abstract}
We present a detailed analysis of the star cluster population in the starburst galaxy NGC 5253. Our work is based on HST optical and VLT $K$ s images. We detect more than 300 clusters, and for all of them we derive the photometry in the $V, I$ and $K \mathrm{~s}$ bands and built a color-color $V-I / V-K$ s diagram. After correction for star contamination, we matched 115 sources in all the three bands $V, I$ and $K$ s. Comparison of the data points with models shows that most of the sources are affected by a red excess. The ages of the younger clusters are obtained from an HST H $\alpha$ image which makes it possible to partially remove the degeneracy between age and extinction and to calculate the screen optical depth toward the clusters. The $V$ and $K$ s luminosity functions show a marked turnover at $M_{\mathrm{V}}=-7.3$ and $M_{K}=-10.2$, with a shape roughly similar to the Gaussian distribution of old globular clusters. We convert the luminosity function into a mass function, and find that the clusters younger than $10 \mathrm{Myr}$ show a power law distribution with slope -1.6 , while the distribution of the older clusters has a maximum at about $4 \times 10^{4} M_{\odot}$.
\end{abstract}

Key words. galaxies: individual: NGC 5253 - galaxies: star clusters - galaxies: starburst

\section{Introduction}

NGC 5253, an irregular dwarf galaxy in the Centaurus Group, is one of the best objects available to study young starbursts. It is in fact one of the closest starburst galaxies, with heliocentric distance of $3.3 \mathrm{Mpc}$ (Gibson et al. 2000), so that $1^{\prime \prime}$ corresponds to 16 parsec. Moreover it is one of the youngest starburst galaxies known (Rieke et al. 1988), with an age likely $\leq 10 \mathrm{Myr}$, as implied by the detection of spectral features arising from Wolf-Rayet stars (Schaerer et al. 1997) and from its almost entirely thermal radio spectrum with very little synchrotron emission from supernova remnants (Beck et al. 1996). Its metallicity is sub-solar and about $Z_{\odot} / 6$ (Kobulnicky et al. 1999).

At centimetric wavelengths, Turner et al. (1998) and Turner et al. (2000) have observed several nebulae, which they derive to be ionized by 200-1000 O stars, suggesting that very large clusters are the preferred mode of star formation in the central regions of the galaxy.

The galaxy hosts hundreds of large and massive star clusters (Caldwell et al. 1989). These objects are believed to be the key features of the most intense star forming episodes (de Grijs 2003a) and have been observed in many starburst galaxies, from the extreme Luminous Infrared Galaxies (e.g. Pasquali et al. 2003) to nearby dwarf irregular galaxies (e.g.

* Based on observations made with the ESO VLT, program 69.B-0345, and on HST observations obtained from the ESO/ST-ECF Science Archive Facility.
Billett et al. 2002; Anders et al. 2004). The properties of these clusters have suggested that at least a fraction of them may eventually be related with the population of old globular clusters observed in more quiescent galaxies (e.g. Boutloukos \& Lamers 2003). However, their mass function, as measured in many young systems, does not show a preferred mass scale as is observed for globular clusters (Fall \& Zang 2001). This indicates that some evolution, for instance evaporation of nongravitationally bound clusters, has to take place. They can also be important as probes of the initial mass function in the extreme environments required for cluster formation, and of the formation and evolution of their host galaxies.

High spatial resolution optical observations with WFPC2 on HST by Calzetti et al. (1997, hereafter C97) have made it possible to resolve with unprecedented detail the central part of NGC 5253 and to carry out a detailed study of the brightest optical clusters and of the dust content of the galaxy. They found that the dust reddening is markedly inhomogeneous across the galaxy's central $20^{\prime \prime}$ region, alternating regions of small and large reddening, so that, even in this relatively low-metallicity galaxy, the early stage of star formation is associated with the formation of dust-embedded clusters. For the six brightest clusters they derived ages spanning a range between 2 and $50 \mathrm{Myr}$. A mass around $10^{5} M_{\odot}$ was claimed for the four oldest clusters (age 10-50 Myr), located south of the central star formation region. Between $20 \%$ and $65 \%$ of the ionizing photons in the northern star-forming region would be produced by the remaining two clusters. For the youngest cluster, named 
cluster-5, C97 derived a mass of about $10^{6} M_{\odot}$, which makes it a super-star cluster candidate, according to the definition by Billett et al. (2002). The ages of the bright clusters in the center of the galaxy seem to be anti-correlated with the amount of dust obscuration, supporting the idea that clusters form deeply embedded in dust. For cluster-5, C97 derived an extinction $A_{\mathrm{V}}>9$ mag.

Harris et al. (2004) have used the same dataset of C97 to select 33 resolved clusters detected in all the filters $F 300 \mathrm{~W}$, $F 547 M$ and $F 814 W$. They have measured aperture photometry in all bands, extinction-corrected using the ratio $\mathrm{H} \alpha$ to $\mathrm{H} \beta$. They derived age and mass estimates for the selected clusters, finding that all their objects were younger than $20 \mathrm{Myr}$, with masses between a few thousand to $10^{5} M_{\odot}$. The young age of all the studied clusters was related to a particularly short dissolution timescale in NGC 5253.

Vanzi \& Sauvage (2004) have observed the galaxy in the infrared and millimeter. They used those observations together with data available in the literature to obtain the spectral energy distribution of cluster-5. From their analysis this cluster turned out to be a super-star cluster with a bolometric luminosity of $1.2 \times 10^{9} L_{\odot}$ and an equivalent O7V star number of 4700 . The optical depth derived toward the cluster is $\tau \sim 7-8$.

Considering the major role of extinction in this galaxy and of dust-enshrouded star clusters for its star formation, we add high-spatial-resolution near-IR observations to the optical dataset used by $\mathrm{C} 97$, in order to sample a wider range of optical depth and to study the properties of the extincted clusters.

After the description of the data set used in Sect. 2, we present in Sect. 3 the results of the photometry of about $600 \mathrm{ob}-$ jects in the $I, V$ and $K$ band. The color-color diagram and the nebular emission are then used in Sect. 4 to derive the extinction of the clusters. The age of the $\mathrm{H} \alpha$-bright clusters is computed from the equivalent width $(E W)$ of the line. Finally, the luminosity and mass functions of the clusters are presented.

\section{Observations and data reduction}

In this work we have used the $K$ s observations of Vanzi \& Sauvage (2004) combined with HST archive data. The archival WFPC2 images (Proposal ID: 6524 and 9144) have the galaxy centered on the WF3 chip, and they are the results of two pairs of exposures, taken with the second pair shifted relative to the first one in order to reject hot pixels and cosmic rays. The $F 814 W$ filter is the equivalent of the $I$ band for WFPC2, while the $F 547 M$ filter was used for the $V$ band. Because of the small recession velocity of the target $\left(404 \mathrm{~km} \mathrm{~s}^{-1}\right.$ ), the $F 656 N$ filter is almost centered on the $\mathrm{H} \alpha$ line; the $F 673 N$ filter is instead used to measure the underlying stellar continuum. This filter contains the $[\mathrm{SII}](6712,6731)$ emission lines but, as in NGC 5253 the ratio $\mathrm{H} \alpha /[\mathrm{SII}]$ is typically larger than 15 (Campbell et al. 1986), we can consider this contribution as negligible for our purpose.

The $F 814 W$ image was obtained from four exposures with $400.0,180.0,400.0$ and $180.0 \mathrm{~s}$ of integration time; the $F 547 M$ image from 600.0, 200.0, 600.0 and 200.0 s exposures; the $F 656 N$ image from $1500.0,500.0,1100.0$ and $500.0 \mathrm{~s}$; for $F 673 N$ only 3 images were available, with exposure times
$1200.0,600.0$ and $600.0 \mathrm{~s}$. The reduction was performed using the On The Fly Reprocessing system of the HST archive (Swam et al. 2001). Our data set is summarized in Table 1.

The absolute photometric calibration of the $K$ s band image was performed with photometric standard stars, while for WFPC2 images the zero points listed in the HST Data Handbook (Baggett et al. 2002) were used. The transformation from the WFPC2 photometric system to $U B V R I$ was performed according to Holtzman et al. (1995), who supply a relation between the HST STMAG system magnitude and the color $(V-I)$ of the target. That relation was inverted to derive the color $(V-I)$ as a function of the observed $(F 547 M-F 814 W)$. The coefficients, which are also functions of the color, were chosen using as initial guess the $(V-I)$ color derived with the synphot package (Simon \& Shaw 1996) for a B5 star with the same $(F 547 M-F 814 W)$. The corrections for the magnitudes were derived from those on the color assuming $(V-F 547 M)=-0.036$. It must be noted that the difference between the $F 814 W$ and the $I$ filter is more than 1 mag, leading to a large color term $(\sim 1.2 \mathrm{mag})$.

The narrow band images in the $F 656 N$ and $F 673 N$ filters were calibrated using the PHOTFLAM keyword in the image header.

\section{Photometry of the clusters and the color-color diagram}

In Fig. 1 we present a three-color image of NGC 5253 obtained by the combination of HST-F547M (blue), HST-F $814 W$ (green) and VLT-Ks (red). If we assume as a first and crude approximation that the red objects in the image are, on average, more extincted than the others, a simple examination of this image tells us that the dust extinction is quite inhomogeneous across the galaxy. Regions of blue and red objects, in fact, appear to be randomly distributed and, besides the well known dust lane, there is no evidence of a regular pattern or structure. A number of likely highly extincted red objects are visible only in the $K$ s band. A more quantitative idea can be obtained from the photometry of the sources and from the analysis of their color-color diagram.

To extract the photometry of the sources we have tested two different methods. In the first one the background was estimated in an annulus of 0.5 around each object, using a constant circular aperture of 0.5 for photometry. This technique provides reliable results only for the brightest and isolated objects (see C97), while the error obtained from a sample of artificial sources of fixed magnitude $m_{\mathrm{V}}=18.4$ is up to about $0.5 \mathrm{mag}$ where the sources are particularly crowded. To improve the photometry of the faint sources we have chosen a different method to estimate and subtract the background which consisted in fitting the luminosity profile of the underlying galaxy. All the sources with more than $2.5 \sigma$ detection were rejected to compute the fit, and Legendre polynomials of order 40, 30 and 28 were used respectively for the $V, I$ and $K$ s band image. The orders chosen were the ones that gave the best fit and minimized the residuals. The obtained fitted surface was then subtracted from the image of the galaxy, and the resulting image was used for the photometry. The error for the photometry 
Table 1. Observations of NGC 5253. Exp. time is the total exposure time (for composite images). FWHM is the Full Width Half Maximum of a point source.

\begin{tabular}{lccccc}
\hline \hline Filter & Instrument & Mean wav. $(\AA)$ & Bandpass $(\AA)$ & Exp. time (s) & $F W H M$ \\
\hline$F 547 M$ & WFPC2 @ HST & 5484.0 & 483.1 & 1600 & $0 . ' 17$ \\
$F 656 N$ & WFPC2 @ HST & 6564.0 & 21.4 & 3600 & 0.17 \\
$F 673 N$ & WFPC2 @ HST & 6732.2 & 21.4 & 2400 & $0^{\prime} 17$ \\
$F 814 W$ & WFPC2 @ HST & 7921.0 & 1488.8 & 1160 & $0 . ' 15$ \\
$K \mathrm{~s}$ & ISAAC @ VLT & 21600 & 2700 & 300 & 0.40 \\
\hline
\end{tabular}

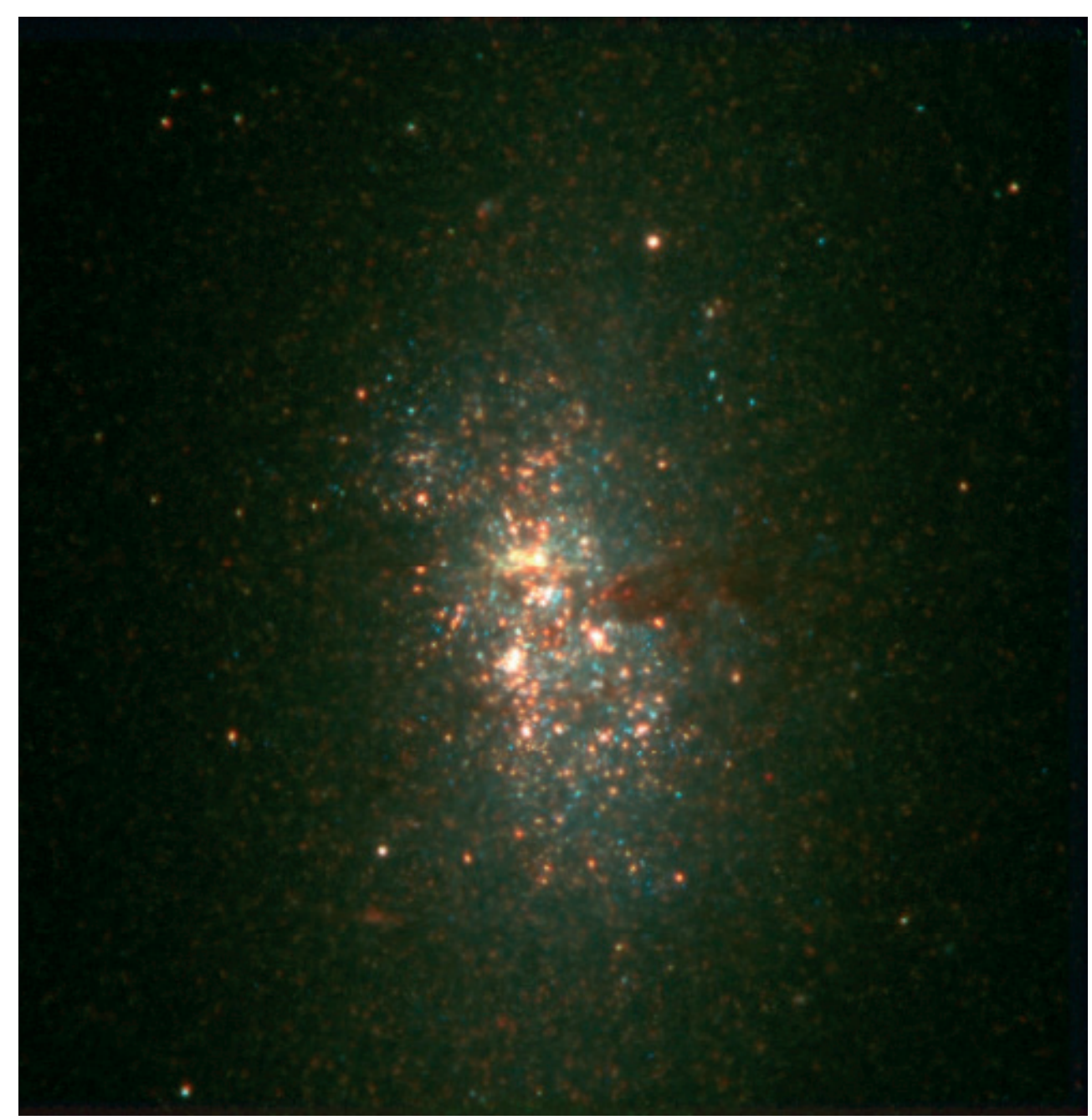

Fig. 1. Three-color image of NGC 5253 in the $V$ band (blue), $I$ band (green) and $K$ s band (red). North is up and east is right. The image is $80^{\prime \prime} \times 80^{\prime \prime}$ in size.

for the same sample of artificial objects is reduced to about $0.05 \mathrm{mag}$.

The detection of the sources was performed using SExtractor (Bertin \& Arnouts 1996) with parameters set to have secure detections, $5 \sigma$ per pixel over an area of 4 pixels. The $V$ and $I$ band images have been previously smoothed, by convolving them with a Gaussian, to reduce them to the angular resolution of the $K$ s band image $(0.4 \operatorname{arcsec})$. In this way it was possible to match multiple sources, resolved only in the HST frames, with a single unresolved source in the ISAAC frame. In the $V$ band smoothed image 491 objects were recovered, 524 in the smoothed $I$ band one, and 357 in the area of the $K$ s band frame covered by the HST observations.

A geometric coordinate transformation between the different images of the galaxy has been computed using as reference 50 bright sources present in all the frames. The transformation was then used to match sources in the three different images. We consider a source as matched in two of the frames when the distance between the centers in the different images, referred to the same coordinate system, is less than 3 pixels (or $0.44)$. With these constraints, 375 sources were matched between the smoothed $V$ and $I$ band frames, and 192 between the $K$ s band and the smoothed $V$ band ones. 178 sources were 
matched in all the three bands. For all other objects, and for the sources matched only in two of the filters, we give an upper or lower limit for the colors, using the $80 \%$ completeness magnitudes for a $5 \sigma$ detection in the frame where the objects are not recovered. This is about 21 for the $V$ and $I$ band and 18 for the $K$ s band.

The photometry of the detected sources was carried out on the background-subtracted images, using a circular aperture of $0.75,1.87$ times the PSF, i.e. 5 pixels in the $K$ s band image and 7.5 pixels in the smoothed $V$ and $I$ band ones.

One of the main sources of error for the photometry is the crowding of the sources. To estimate this contribution we used the sample of clusters detected in the unsmoothed images in each band to calculate the probability $P(L)$ of finding an object of a given luminosity $L$ in the aperture used for photometry, and then we summed all the contributions $L \cdot P(L)$. We ended with photometric errors due to crowding of about $0.12,0.11$, 0.04 mag for the $V, I$ and $K$ band respectively.

The resulting $V-I / V-K$ color-color plot is shown in Fig. 2. The uncertainties in both colors vary between 0.12 to 0.44 mag. The circles in the upper panel are the sources matched in all three bands, the filled ones with a mean of the uncertainties in the two colors of less than $0.2 \mathrm{mag}$ and the open ones with errors between 0.2 and $0.44 \mathrm{mag}$. The arrows in the lower panel are upper and lower limits on the colors for the objects detected in $V$ and $I$ but not in $K$ s (the ones pointing to the left) or in $I$ and $K \mathrm{~s}$ but not in $V$ (pointing to the upper right). The screen extinction vector for $A_{\mathrm{V}}=2$ is also reported. The solid line is the prediction of the Starburst99 model (SB99, Leitherer et al. 1999) for an instantaneous burst with metallicity $Z=1 / 5 Z_{\odot}$, assuming a Salpeter IMF with $\alpha=2.35, M_{\text {low }}=1 M_{\odot}$ and $M_{\text {up }}=100 M_{\odot}$, with an age coverage $10^{5}-10^{9} \mathrm{yr}$, while the dot-dashed line is the same model but with $Z=Z_{\odot}$.

\section{Analysis and discussion}

From a comparison of the data with the models it is evident that a large number of sources is affected by a significant red excess. The degeneracy between age and extinction, both producing a reddening of the clusters, prevents us from determining these two quantities independently using the color-color diagram alone. This degeneracy will be partly removed in Sect. 4.2, and the derived ages will be used to evaluate the extinction toward the clusters in Sect. 4.3. This will enable us to build the luminosity and finally the mass function in Sects. 4.4 and 4.5 respectively.

But first of all, note that there is a significant difference between the solar and sub-solar models. The first extends much further in the red mainly because of the red supergiant phase between 5 and $15 \mathrm{Myr}$ of age, this phase being artificially suppressed in the sub-solar model. In fact the sub-solar models fail to reproduce the right number of red supergiants, and there are indeed indications that their number increases rather than decreases as the metallicity is reduced (e.g. Maeder \& Meynet 2001; Eggenberger et al. 2002, and references therein). Moreover, the duration of the red phase is expected to increase as the abundance decreases (Maeder \& Meynet 2001). In other words, neither model can be considered a perfect reference, as
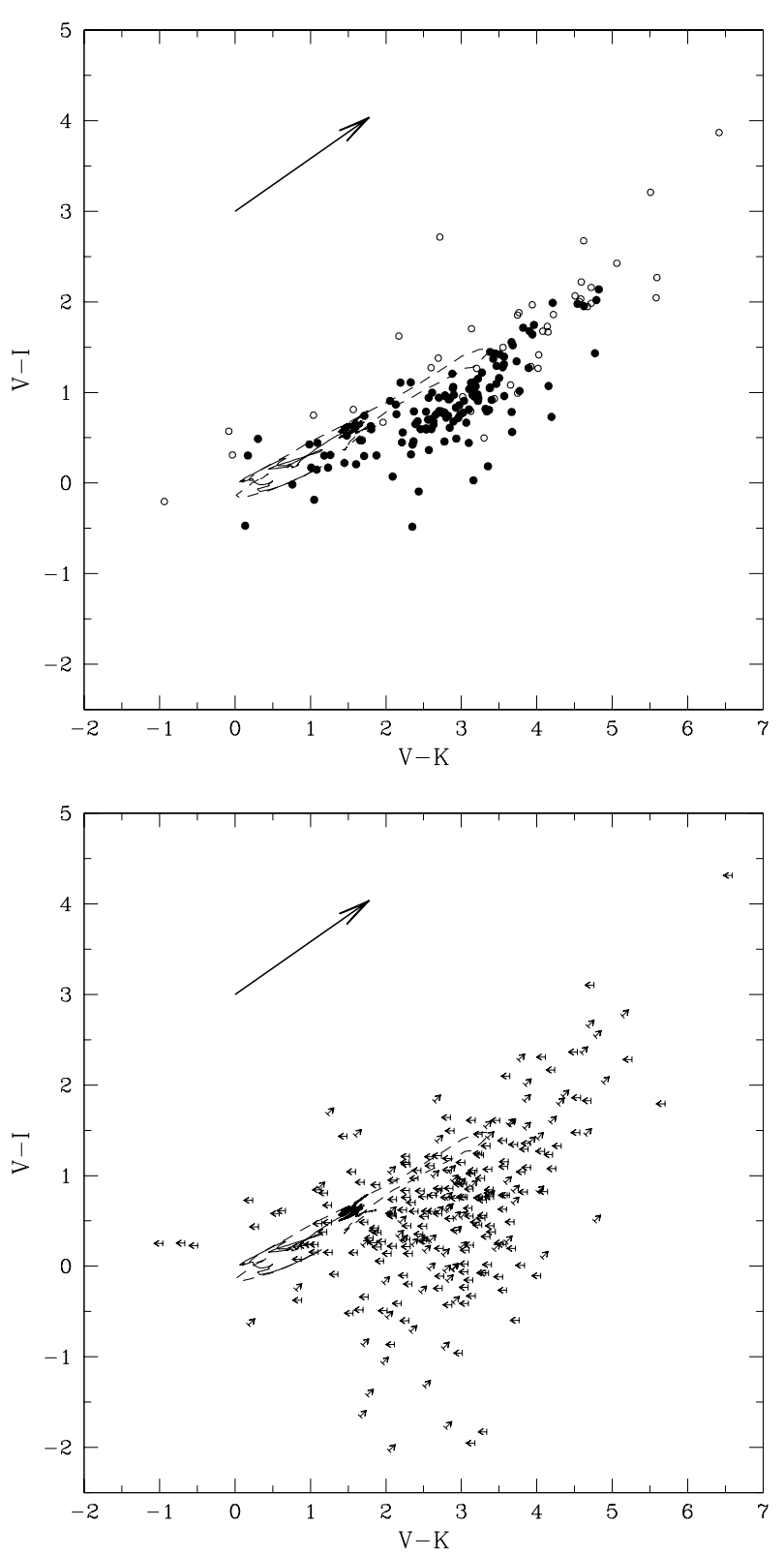

Fig. 2. Color-color plot of the observed clusters in NGC 5253. Upper panel: the circles are the clusters detected in all all bands $(V, I$, and $K \mathrm{~s}$ ), the filled ones with a mean of the uncertainties in the two colors of less than 0.2 mag and the open ones with errors between 0.2 and $0.44 \mathrm{mag}$. The solid line is the SB99 model of an instantaneous burst of star formation, with $Z=0.004$, assuming a Salpeter IMF with $\alpha=2.35$ and $M_{\text {up }}=100 M_{\odot}$, while the dot-dashed line is the same model but with $Z=Z_{\odot}$, where the red supergiants loop is evident (see text). The arrow in the upper left corner is the screen extinction vector for $A_{\mathrm{V}}=2$ mag. Lower panel: the arrows are upper and lower limits on the colors for the objects detected in $V$ and $I$ but not in $K$ s (the ones pointing to the left) or in $I$ and $K$ s but not in $V$ (pointing to the upper right). The SB99 models and the screen extinction vector are plotted as in the upper panel.

this effect has important consequences for the predicted colors (Origlia et al. 1999). In the following we will consider both models, comparing the results obtained in the two cases. 


\subsection{Star contamination}

Since most of the sources detected are barely resolved or not at all, our sample could be significantly contaminated by stars, both foreground and galaxy members. Foreground stars should not be a source of confusion, as the standard Milky Way star count models (e.g. Ratnatunga \& Bachall 1985) predict roughly 2-3 foreground stars in our field of view with $m_{\mathrm{V}}<21$. However, individual blue and red supergiant stars can have absolute magnitudes as bright as $M_{\mathrm{V}} \simeq-9$ (Humphreys 1983), that is $m_{\mathrm{V}} \simeq 18.6$ at the distance of NGC 5253. As a consequence, our cluster sample can be contaminated by single bright stars.

As only few of the sources in NGC 5253 are brighter than $M_{\mathrm{V}}<-9$, we used the high angular resolution provided by the WFPC2 images to distinguish the resolved clusters from the point like objects. The archive WFPC2 images were centered on the WF3 chip, which provides a PSF with FWHM $\sim 0$ ' 13 , corresponding to $\sim 2 \mathrm{pc}$ at the distance of the galaxy. This is slightly smaller than the typical dimensions of young star clusters, so that also in the WF images clusters are in general only barely resolved. The $F W H M$ of the sources matched in the three bands was measured in the $F 547 M$ original image and compared with the PSF provided by TinyTim for the same configuration as well as with the PSF of bona-fide stars in the field. Objects whose PSF was indistinguishable from point sources, i.e. with $F W H M<0$ '. 153 , were then discarded from the following analysis. This way we removed from the sample $\sim 35 \%$ of the 178 sources matched in all the filters, ending up with 115 clusters.

\subsection{The ages of the clusters}

As already mentioned, the ages of the clusters cannot be derived in a unique way from the colors, so that it is essential to have an independent age indicator. The Equivalent Width $(E W)$ of the $\mathrm{H} \alpha$ emission line can be used for this purpose, as it decreases monotonically with age, according to the SB99 model, with little dependence on metallicity. Therefore we used this method to derive the ages of 51 clusters matched in $V, I$ and $K \mathrm{~s}$ which have also been detected in $\mathrm{H} \alpha$; the ages are obtained by comparison of the measured $E W$ with the SB99 model prediction. The derived ages span a range between 3 and 19 Myr.

According to the model, older clusters are not detectable in the $\mathrm{H} \alpha$ image because of their faint emission, since very few $\mathrm{O}$ and $\mathrm{B}$ stars are present to ionize the gas. To check that young faint objects, with low flux but large $E W$, were not placed in the sample of old objects, we derive an estimate of the flux expected in the $F 656 N(\mathrm{H} \alpha)$ filter for all the objects in the sample. The flux in the $\mathrm{H} \alpha$ continuum, $F 673 N$ filter, was evaluated for the objects matched in the $V$ and $I$ bands by linear interpolation between the fluxes measured in these two bands. In this way it is possible to calculate the expected flux in $\mathrm{H} \alpha$ for all the objects as a function of the $E W$. If we assume an $E W>45 \AA$, corresponding to an age of $10 \mathrm{Myr}$ according to the SB99 model, the derived $\mathrm{H} \alpha$ flux is above our detection limit for all the objects, so that we can state that we are not missing any source with $E W$ greater than $45 \AA$ and younger than 10 Myr. Therefore

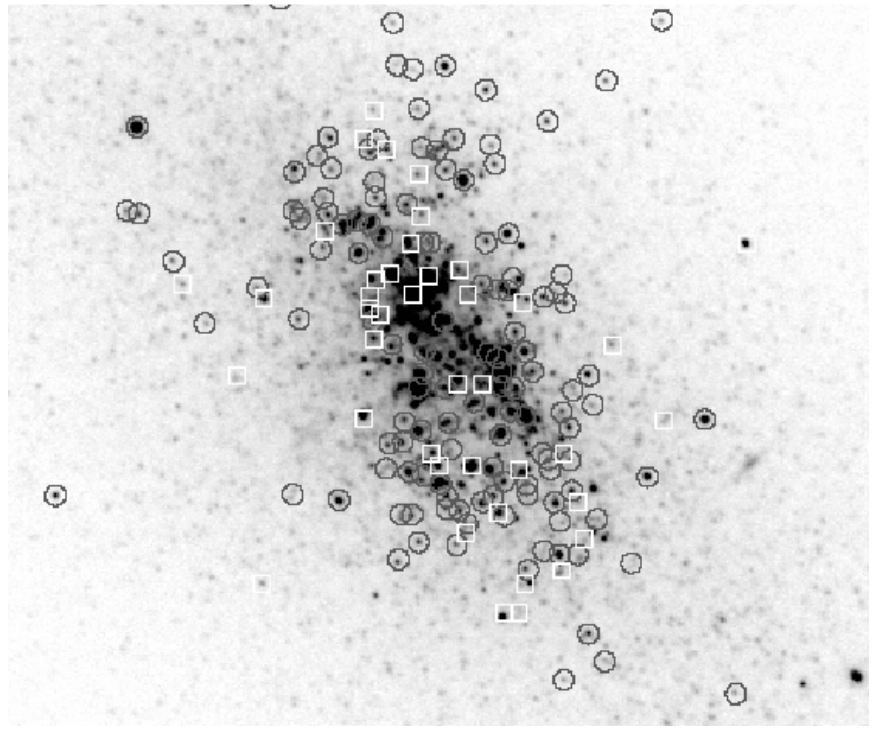

Fig. 3. Spatial distribution of older (gray circles, age $>20 \mathrm{Myr}$ ) and younger (white rectangles, age $<20 \mathrm{Myr}$ ) matched clusters, compared with the VLT $K$ s band image.

our age estimation provides reliable values for "young" clusters detected in $\mathrm{H} \alpha$, and a lower limit of $10 \mathrm{Myr}$ for the "old" ones, yielding a division of the sources in two subsections. In Fig. 3 we plot the spatial distribution of "old" (grey circles) and "young" (white squares) clusters. No clear correlation is present between the spatial distribution of the clusters in the galaxy and their ages, although there is a group of young objects located at the position of cluster-5.

With this constraint on the age, it is possible to derive the extinction by the color excess once the SB99 model is assumed, as explained in the following section.

\subsection{Extinction and dust}

We derived the extinction using our color-color diagram and the color excess of the clusters with respect to the SB99 model, once the age of each source is known. We assume that the discrepancy between the model and the data points is produced by the effect of screen extinction and emission by dust. If we assume a dust temperature of $1000 \mathrm{~K}$, its emission contributes significantly only in the $K$ band, and as a consequence moves the points to the right, increasing $V-K$. Combining these two effects, it is possible to derive the extinction for the clusters of known age, for which we know the theoretical position in the diagram. Visual screen extinctions in the range 0 to $7.5 \mathrm{mag}$ were found, with an average of $1.6 \mathrm{mag}$. An estimate of the extinction for the "old" clusters can be obtained assuming a reference age of $20 \mathrm{Myr}$ for all these objects. This is obviously a crude approximation; however, the change of colors with age is much smaller for clusters older than $20 \mathrm{Myr}(\Delta(V-I) \sim 0.2$ between $20 \mathrm{Myr}$ and $1 \mathrm{Gyr})$ than for younger ages $(\Delta(V-I) \sim 1.9$ between 0 and $20 \mathrm{Myr})$. This way we obtain extinctions in the same range as for the young clusters.

Note that in using the color diagram to derive the screen extinction we are underestimating the actual column density of 

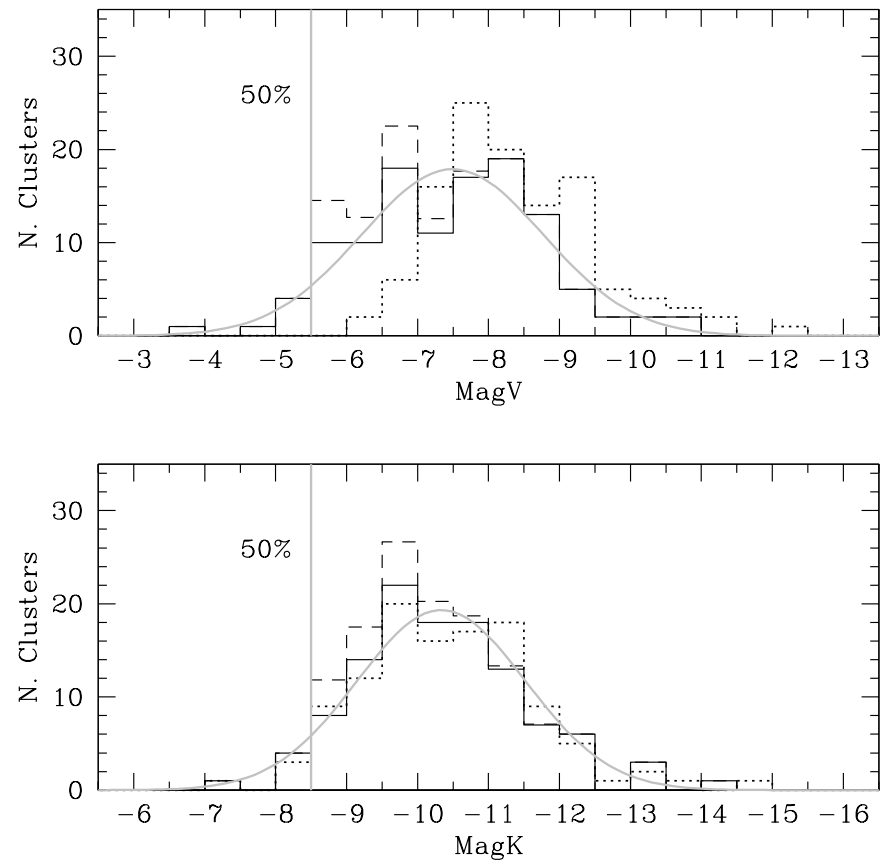

Fig. 4. The luminosity function of the sources observed in $V$ and $K \mathrm{~s}$ band. The solid line is the observed function, and the dotted one is after correction for extinction. The dashed line is the completenesscorrected function, where the correction is applied to the $50 \%$ completeness limit. The Gaussian curves are the best fit to the observed distributions.

obscuring material, as we are neglecting the scattering by dust grains, which strongly affects colors. In fact, while absorption reddens the intrinsic spectrum, scattering makes it bluer by reflecting some light in our direction (e.g. Witt et al. 1992). The net result is that the magnitude of the extinction decreases for a given optical depth when scattering is taken into account. In particular, Vanzi \& Sauvage (2004) find that the extinction is 3-4 times higher than in the simple screen case when the effect of scattering is included for the extreme case of cluster 5 .

The X-ray and radio data available in the literature could not be used to measure the extinction, as none of the objects detected at those wavelengths can be clearly associated with optical or NIR sources (Turner et al. 1998; Strickland \& Stevens 1999; Summers et al. 2004).

\subsection{The luminosity function}

In Fig. 4 we show the $V$ and $K$ s band luminosity functions of the selected sources. The solid line is the observed function, while the dotted one is after correction for extinction. As expected, the effect of the extinction is much more relevant in $V$ than it is in $K$ s.

Completeness tests were performed in, for both the $V$ band and $K \mathrm{~s}$ band images adding in each frame 80 artificial objects, concentrated in the central part of the galaxy, with shapes derived by using isolated, high $\mathrm{S} / \mathrm{N}$ clusters from the same image. The sources were added each time with different magnitudes, and recovered with the same technique used for the real clusters. The catalogue of the recovered sources was then cross-correlated with the catalogue of the added artificial sources to derive the fraction of recovered objects for each luminosity channel, and thus the completeness correction. The dashed line in Fig. 4 is the luminosity function with the completeness correction obtained from these simulations. The correction is applied to the $50 \%$ completeness limit magnitude, where half of the artificial objects were recovered. The two corrections, for extinction and for completeness, cannot be combined to produce the intrinsic luminosity function. In addition, the luminosity function was not corrected for age effects, as we have only a lower limit for clusters not detected in $\mathrm{H} \alpha$.

The luminosity functions show a peak about $1.5 \mathrm{mag}$ brighter than our $50 \%$ completeness limit, both in the $V\left(M_{\mathrm{V}}\right.$ (peak) $\left.=-7.5\right)$ and the $K \mathrm{~s}$ band $\left(M_{K}\right.$ (peak) $=$ $-10.3)$. The significance of this peak is highest in the $K$ band where the effect of the extinction is minimum. The luminosity distribution $\psi(L)$ is remarkably close to the shape of the old globular cluster luminosity function (roughly Gaussian with the peak or turnover magnitude at $M_{\mathrm{V}} \simeq-7.4$, e.g. de Grijs et al. 2003b), but quite different from the power law shape $\left(\psi(L) T l \propto L^{-\alpha} \mathrm{d} L\right)$ derived by Whitmore et al. (1999) for the luminosity function of young (age $<100 \mathrm{Myr}$ ) clusters in the Antennae galaxy.

A systematic underestimate of the completeness correction would be needed to obtain a power law from the observed distribution. This cannot be completely ruled out, especially below the $80 \%$ completeness limit, which corresponds to the detected peak.

\subsection{The mass function}

With all the information gathered about the star cluster population in NGC 5253, it is possible to provide, under a few assumptions, an estimate of the mass function of these objects. To derive the mass we will use here the $K$ s luminosity as it is less affected by the extinction and is a better tracer of the mass.

To convert the $K$ s luminosity into mass we have to assume a $K$ s-to-mass ratio. Vanzi \& Sauvage (2004) have studied in detail the spectral energy distribution (SED) of Cluster-5 using all data available in the literature, and developed a model of both the stellar cluster and the surrounding dust shell. Here we assume that the shell geometry of cluster and dust, the dust properties and the stellar SED of Cluster-5 are typical of the sources in NGC 5253. Under this assumption, we have run a set of models at different ages and with different amounts of optical opacity, and therefore of dust, in order to obtain the light-tomass ratio, or the ratio between the $K$ luminosity and the total mass. The model tabulates the $K$ luminosity to cluster mass ratio for a range of ages and extinction. For each observed cluster we use our determined age and extinction to select the most appropriate model and use the corresponding light-to-mass ratio to derive the cluster mass.

This way it is possible to convert the luminosity function into a mass function. We have done this using both the solar (dotted) and sub-solar (solid) models to derive the screen extinction. The results are shown in Fig. 5. The upper panel shows the mass function of the sources younger than $20 \mathrm{My}$ detected 

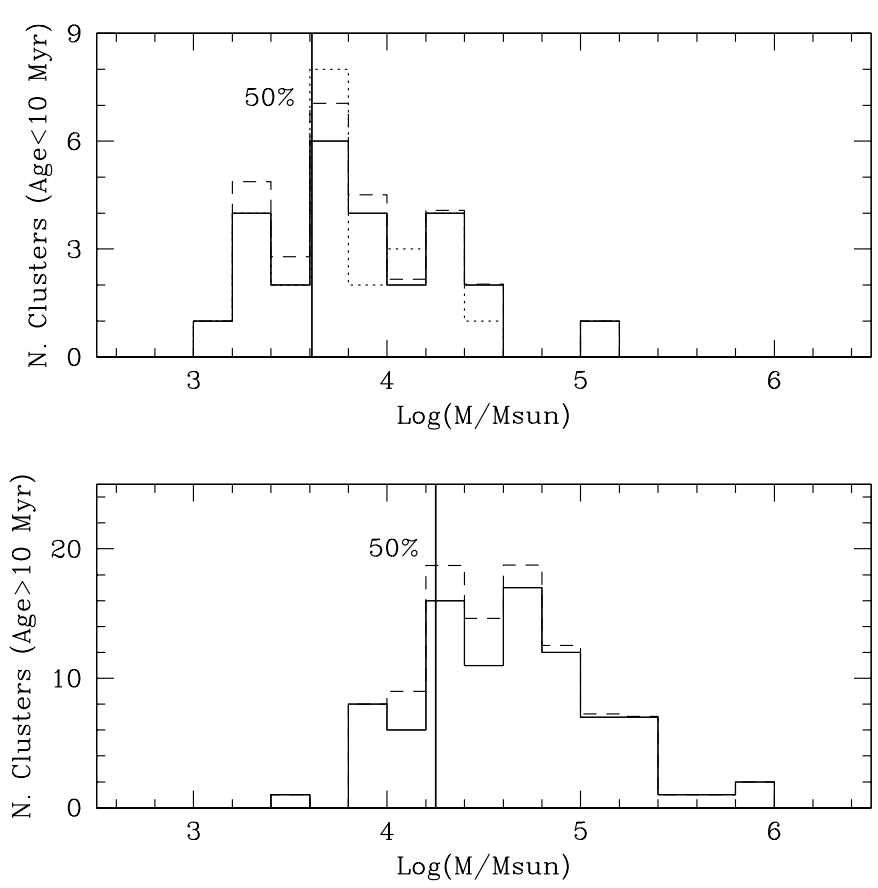

Fig. 5. Mass function of the young clusters detected in $\mathrm{H} \alpha$ (upper panel) and of the older ones (lower panel). The 50\% completeness limits are marked by vertical lines. The dotted line in the upper panel uses the solar model as reference, while the dashed lines are the mass functions after the completeness correction has been applied.

in $\mathrm{H} \alpha$ obtained using the solar and sub-solar models as reference. The difference between the two is not significant, as few of the clusters have an age corresponding to the supergiant phase, where the loop distinguishing the two models is located. The lower panel shows the mass function of the clusters not detected in $\mathrm{H} \alpha$ and therefore older than $10 \mathrm{Myr}$, assuming a reference age of $20 \mathrm{Myr}$ in this case. The dashed lines are the mass functions after the completeness correction has been applied. The same number and luminosity of missed clusters derived from the correction of the luminosity function was used to compute the completeness correction for the mass function. Their mass was calculated from the luminosity assuming the average extinction of the observed clusters $\left(A_{\mathrm{V}}=0.98 \mathrm{mag}\right)$, the average age of $8 \mathrm{Myr}$ of the young clusters and a reference age of 20 Myr for the older ones.

In the distribution of the old objects a turnover is detectable at about $5 \times 10^{4} M_{\odot}, 2.5$ times more massive than the $50 \%$ completeness limit. In the distribution of the young objects no peak is observable above the completeness limit, and the data are well reproduced by a power law. The best fitting power law index for the mass function $\psi(M) \mathrm{d} M \propto M^{-\beta} \mathrm{d} M$ of the young objects is $\beta=1.56$, while $\beta=1.82$ is obtained for objects more massive than the peak in the old cluster distribution.

Using a selection criterion between clusters and single bright stars based only on the TinyTim model PSF for the $V$ band image, instead of using stars in the field, we discard as unresolved sources around $15 \%$ of the objects matched in all the three bands. In this case we obtain a slightly larger exponent for the young cluster distribution, $\beta=1.71$, while the peak in the luminosity and and mass functions of old clusters is still present. The power law index $\beta$ derived for the mass function of younger clusters is in the same range as what has been found in a wide variety of galaxies (e.g. de Grijs et al. 2003d), and fully consistent with the mass function index of clumps in a large number of molecular clouds (see e.g. Kramer et al. 1998, and references therein).

The currently most popular model for the dynamical evolution of star clusters predicts that the power law mass and luminosity functions of young star clusters, detected in many nearby galaxies (see Whitmore et al. 1999; and Zhang \& Fall 1999, for the "Antennae" galaxies), will be rapidly transformed into the Gaussian functions of old globular clusters, under the action of several effects (e.g. Fall \& Zhang 2001). These include the preferential depletion of low-mass clusters, by evaporation due to two-body relaxation and by tidal interactions with the gravitational field of the host galaxy, and the preferential disruption of high-mass clusters by dynamical friction. Till now there was only one example of a turnover in the mass and luminosity distribution for intermediate-age ( $\sim 1 \mathrm{Gyr})$ clusters, reported by de Grijs et al. (2003c) in the center of M 82.

Our data may be consistent with a scenario where young, power-law-distributed clusters are superimposed on an older population with the same initial power law index that has already developed a turnover in the luminosity and mass distributions. Comparing the total mass of the youngest $\left(4.1 \times 10^{5} M_{\odot}\right)$ to the total mass of the oldest clusters $\left(7.4 \times 10^{6} M_{\odot}\right)$, we find that the most recent burst of star formation is at least $\sim 20$ times less intense than the previous ones.

The total mass of the matched clusters is $7.8 \times 10^{6} M_{\odot}$, which has to be compared with the total mass of the galaxy of $\sim 6 \times 10^{9} M_{\odot}$ (Vanzi \& Sauvage 2004).

\section{Summary and conclusions}

The conclusions of our work can be summarized as follows:

1. We have detected a large number of compact sources in the starburst galaxy NGC 5253 and built their $V-I / V-$ $K$ s color-color diagram. From the comparison of the data points with models we found that most sources are affected by a significant color excess, which we mostly ascribe to dust.

2. 178 sources were matched in all the three bands $V, I$, and $K$. To take into account the stellar contamination by single bright stars we have discarded on the basis of their pointlike profiles around $35 \%$ of these sources, ending up with 115 clusters.

3. We have partially broken the age-extinction degeneracy of the color-color diagram and derived the luminosity and mass functions of the clusters.

4. The ages of the clusters, as measured from their $\mathrm{H} \alpha$ emission, span a range from few to 19 Myr. Many of the clusters are not detected in $\mathrm{H} \alpha$, implying ages $>10 \mathrm{Myr}$.

5. The clusters younger than $20 \mathrm{Myr}$ show a mass function that can be modeled with a power law $\psi(M) \propto M^{-\beta}$, with an exponent $\beta=1.6$. For objects more massive than $5 \times 10^{4} M_{\odot}$ in the older cluster distribution a value of $\beta=1.8$ is obtained. These values are in the same range as what has been 
found in a wide variety of galaxies, including interacting galaxies and more quiescent objects (de Grijs et al. 2003d), and fully consistent with the mass function index of clumps in a large number of molecular clouds (Kramer et al. 1998).

6. Both the luminosity functions and the mass function of the oldest clusters show a peak or turnover above our $50 \%$ completeness limit. The luminosity functions in the $V$ and $K$ s band, in particular, show a Gaussian profile peaked at $M_{\mathrm{V}}=-7.5$ and $M_{K}=-10.3$. The shape of the distribution is remarkably close to that of the old globular clusters, roughly Gaussian with a peak at $M_{\mathrm{V}} \simeq-7.4$, and quite different from the power law distribution found in other young cluster populations. If the peak in our data is confirmed, this galaxy may provide a second example of a turnover in an intermediate age cluster population maybe even younger than in $\mathrm{M} 82$.

Acknowledgements. We want to thank the anonymous referee for useful comments and suggestions. We are also grateful to Ian Stevens for providing us with results of his work before publication. G.C. acknowledges support from the European Southern Observatory during his visit to Chile.

\section{References}

Anders, P., de Grijs, R., Fritze-v. Alvensleben, U., \& Bissantz, N. 2004, MNRAS, 347, 17

Baggett, S., et al. 2002, in HST WFPC2 Data Handbook, v. 4.0, ed. B. Mobasher, Baltimore, STScI

Beck, S. C., Turner, J. L., Ho, P. T. P., Lacy, J. H., \& Kelly, D. M. 1996, ApJ, 457, 610

Bertin, E., \& Arnouts, S. 1996, A\&AS, 117, 393

Billett, O. H., Hunter, D. A., \& Elmegreen, B. G. 2002, AJ, 123, 1454

Boutloukos, S. G., \& Lamers, H. J. G. L. M. 2003, MNRAS, 338, 717

Caldwell, N., \& Phillips, M. M. 1989, ApJ, 338, 789

Calzetti, D., Meurer, G. R., Bohlin, R. C., et al. 1997, AJ, 114, 1834

Campbell, A., Terlevich, R., \& Melnick, J. 1986, MNRAS, 223, 811

de Grijs, R. 2003a, Astron. Geophys., 44, 14

de Grijs, R., Fritze-v. Alvensleben, U., Anders, P., et al. 2003b, MNRAS, 342, 259 de Grijs, R., Bastian, N., \& Lamers, H. J. G. L. M. 2003c, ApJ, 583, L17

de Grijs, R., Anders, P., Bastian, N., et al. 2003d, MNRAS, 343, 1285

Devillard, N. 2001, ASP Conf. Ser., Astronomical Data Analysis Software and Systems X, 10, 238, 525

Eggenberger, P., Meynet, G., \& Maeder, A. 2002, A\&A, 386, 576

Fall, S. M., \& Zhang, Q. 2001, ApJ, 561, 751

Gibson, B. K., Stetson, P. B., Feedman, W. L., et al. 2000, ApJ, 529, 723

Harris, J., Calzetti, D., Gallagher, J. S., Smith, D. A., \& Conselice, C. J. 2004, ApJ, 603, 503

Holtzman, J. A., Burrows, C. J., Casertano, S., et al. 1995, PASP, 107, 1065

Humphreys, R. M. 1983, ApJ, 269, 335

Kobulnicky, H. A., Kennicutt, R. C., \& Pizagno, J. L. 1999, ApJ, 514, 544

Kramer, C., Stutzki, J., Rohrig, R., \& Corneliussen, U. 1998, A\&A, 329,249

Johnson, H. L. 1966, ARA\&A, 4, 193

Leitherer, C., Schaerer, D., Goldader, J. D., et al. 1999, ApJS, 123, 3

Maeder, A., \& Meynet, G. 2001, A\&A, 373, 555

Meurer, G. R., Heckman, T. M., Leitherer, C., et al. 1995, AJ, 110 , 2665

Origlia, L., Goldader, J. D., Leitherer, C., Schaerer, D., \& Oliva, E. 1999, ApJ, 514, 96

Pasquali, A., de Grijs, R., \& Gallagher, J. S. 2003, MNRAS, 345, 161

Ratnatunga, K. U., \& Bahcall, J. N. 1985, ApJS, 59, 63

Rieke, G. H., Lebofsky, M. J., \& Walker, C. E. 1988, ApJ, 325, 679

Schaerer, D., Contini, T., Kunth, D., \& Meynet, G. 1997, ApJ, 481, L75

Simon, B., \& Shaw, R. A. 1996, ASP Conf. Ser., 101, Astronomical Data Analysis Software and Systems V, 5, 183

Strickland, D. K., \& Stevens, I. R. 1999, MNRAS, 306, 43

Summers, L. K., Stevens, I. R., Strickland, D. K., \& Heckman, T. M. 2004 [arXiv: astro-ph/0403669]

Swam, M. S., Hopkins, E., \& Swade, D. A. 2001, ASP Conf. Ser., 238, Astronomical Data Analysis Software and Systems X, 10, 291

Turner, J. L., Ho, P. T. P., \& Beck, S. C. 1998, AJ, 116, 1212

Turner, J. L., Beck, S. C., \& Ho, P. T. P. 2000, ApJ, 532, L109

Vanzi, L., \& Sauvage, M. 2004, A\&A, 415, 509

Whitmore, B. C., Zhang, Q., Leitherer, C., et al. 1999, AJ, 118, 1551

Witt, A. N., Thronson, H. A., \& Capuano, J. M. 1992, ApJ, 393, 611

Zhang, Q., \& Fall, S. M. 1999, ApJ, 527, L81 